\title{
The American Association for Thoracic Surgery 2016 Ethics Forum: Cost-effectiveness and the ethics of left ventricular assist device therapy
}

\author{
John W. C. Entwistle III, MD, PhD
}

From the Division of Cardiothoracic Surgery, Department of Surgery, Thomas Jefferson University, Philadelphia, $\mathrm{Pa}$.

Read at the 96th Annual Meeting of The American Association for Thoracic Surgery, Baltimore, Maryland, May 14-18, 2017.

Received for publication Aug 16, 2016; revisions received March 13, 2017; accepted for publication March 24, 2017; available ahead of print May 5, 2017.

Address for reprints: John W. C. Entwistle III, MD, PhD, Department of Surgery, Thomas Jefferson University, 1025 Walnut St, Suite 607, Philadelphia, PA 19107 (E-mail: John.entwistle @ jefferson.edu).

J Thorac Cardiovasc Surg 2017;154:1315-8

$0022-5223 / \$ 36.00$

Copyright (C) 2017 by The American Association for Thoracic Surgery

http://dx.doi.org/10.1016/j.jtcvs.2017.03.121

Left ventricular assist devices (LVADs) are the standard for many patients with end-stage cardiac failure. Improved quality of life (QOL) and survival come at the expense of ventricular assist device (VAD)-specific complications and stress on the family and caregivers. The ethics of patient selection remain unclear for this expensive therapy that offers limited benefit. In the United States, there are strict medical criteria for permanent LVAD support. However, unlike the situation for heart transplantation, exclusion criteria are not well delineated. This has the potential to produce inconsistent distribution of LVAD therapy and the ethical implications that ensue.

The framework for bioethics put forth by Beauchamp and Childress ${ }^{1}$ includes 4 nonhierarchical principles. Autonomy, beneficence, nonmaleficence, and justice carry equal weight when assessing the ethical course of action. When 2 or more of the principles are in conflict, the appropriateness of an action is determined from the relative good and harm of the individual principles in question. Analysis of these 4 principles is necessary when examining the ethics of the distribution of LVAD therapy.

Under the principle of autonomy, a patient has the right to choose their plan of care. LVAD candidates are facing death from heart failure, the imminence of which is determined by the degree of illness. When a patient is bed-bound with congestive heart failure, they will almost always opt for an LVAD ${ }^{2}$ because they feel they have "no choice." In this situation, autonomy is not the deciding factor to determine whether a patient should be offered an LVAD.

Beneficence, or acting to relieve suffering or lengthen life, usually is fulfilled with LVAD therapy. LVADs can provide years of life compared with medical therapy, and there is a significant and durable improvement in QOL. ${ }^{3}$ Even patients at high risk for mortality are likely to benefit if they survive the initial hospitalization. Beneficence strongly favors LVAD therapy in patients at reasonable operative risk, but it is less clear that this principle favors an LVAD

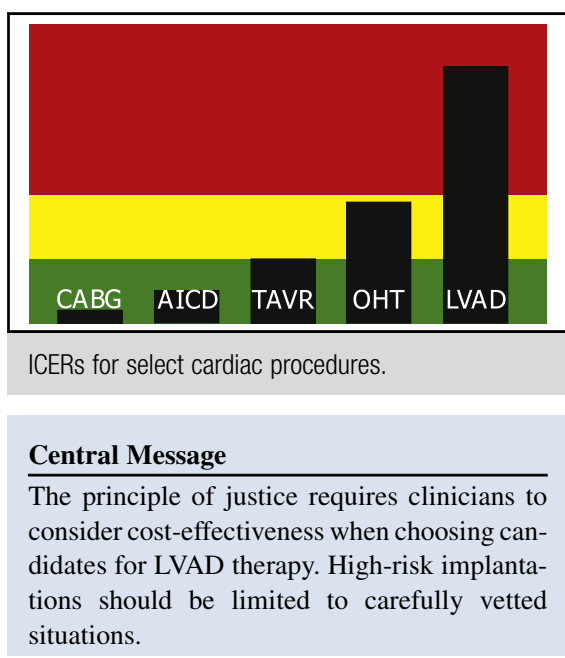

See Editorial Commentary page 1319.

when the chance of survival is low and the chance of complications is high.

Nonmaleficence requires that the physician avoids excessive risk to minimize the chance of producing harm. In LVAD candidates at higher risk, hospital mortality rates approach $20 \%$, and many of these patients will experience significant complications. Acceptance of high rates of morbidity and mortality may be reasonable when the other principles favor implantation, but this should not be the norm because a high complication rate would violate the principle of nonmaleficence.

The principle of justice requires that all patients be treated fairly without bias. Although the other principles are often viewed as patient-centric, justice requires a larger view. When faced with a scare resource, such as an organ for transplantation, justice requires that all transplant candidates have an equal opportunity to receive that organ. A different view of justice may be more appropriate in the case of an unlimited but expensive therapy such as an LVAD. Under this view, justice requires that all persons have an equal opportunity to receive adequate healthcare. The use of a large amount of resources for a single patient may affect the availability of resources necessary to treat other patients. Justice is only served when the implantation of an LVAD does not consume excessive resources.

The principles of autonomy and beneficence favor LVAD implantation most of the time, whereas nonmaleficence 


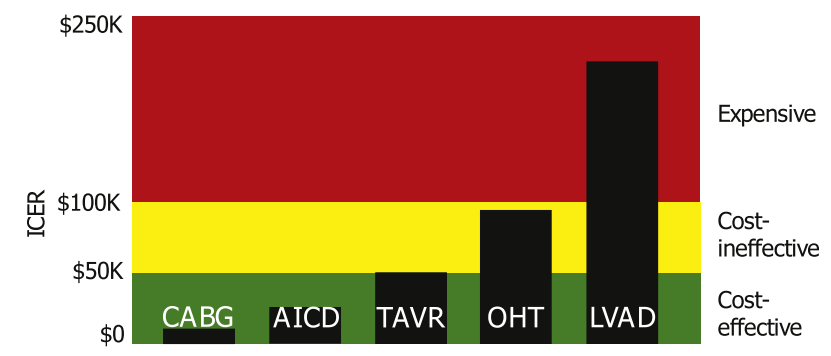

FIGURE 1. ICER for select cardiac procedures with assessment of costeffectiveness by accepted criteria. ICER, Incremental cost-effectiveness ratio; $C A B G$, coronary artery bypass grafting; $A I C D$, automatic implantable cardioverter-defibrillator; TAVR, transcatheter aortic valve replacement; $O H T$, orthotopic heart transplantation; $L V A D$, left ventricular assist device.

favors an LVAD in the absence of elevated risk. However, when risk is sufficiently high such that the chance of benefit is low, beneficence is less clear and justice may help lead to the most ethical action.

Consider 2 patients in class IV heart failure with cardiogenic shock, identical except for age. They have reasonable family support, yet neither is an ideal transplant candidate. The first patient is 40 years old, and the second patient is 70 years old. Either would likely require short-term support before a long-term device. In each case, is LVAD implantation ethically appropriate? Is it ethically reasonable to make a different decision for each patient? How is the decision made to proceed with LVAD or continue medical management?

Unlike heart transplantation, device availability is not a factor. The cost of implantation is not an adequate factor either, because many expensive therapies such as transplantation are widely accepted. Effectiveness of the therapy also is not appropriate, because LVADs are effective in prolonging life and improving QOL regardless of age or medical condition. Even with a $10 \%$ chance of survival, one can make the argument that such a low rate of success is effective when compared with certain death.

A commonly used standard in this situation is costeffectiveness. The incremental cost-effectiveness ratio (ICER) is the difference in cost of 2 treatments divided by the difference in quality-adjusted life years (QALYs) that each produces. A procedure with an ICER of $\$ 10,000$ costs $\$ 10,000$ per QALY gained over standard therapy. In general, therapies with an ICER less than $\$ 50,000$ are considered cost-effective, and those that are significantly higher are cost-ineffective (Figure 1). Some argue that therapies with an ICER between $\$ 50,000$ and $\$ 100,000$ or more are expensive yet may be accepted as cost-effective. ${ }^{4}$ In patients facing death, an end-of-life premium is sometimes invoked, ${ }^{5}$ whereby the cost-effectiveness threshold is raised for therapies when death is imminent, justifying an ICER up to $\$ 100,000$ or more. The cost-effectiveness of LVAD therapy is critical in determining the justice in offering LVAD support.
The pulsatile HeartMate device (Thoratec Corp, Pleasanton, Calif) used in the REMATCH Trial had an ICER of $\$ 802,700,{ }^{6}$ whereas the ICER for newer devices is approximately $\$ 200,000$ when compared with optimal medical management. $^{7-9}$ Despite exceeding the usual costeffective criteria, implantations are covered by insurance and continue at a rapid rate. Is it possible for LVADs to be cost-effective under the threshold of $\$ 50,000$ to $\$ 100,000 /$ QALY? The answer is "possibly," but only with very careful patient selection. The ICER can be lowered through longer survival and fewer costly complications. Patients who receive an implant before inotropes are necessary have a 3-year survival in excess of $90 \%$ compared with $50 \%$ when they undergo urgent operation. ${ }^{10}$ The costs of implantation were nearly twice as high in patients who died during the index hospitalization compared with those who survived, and costs were 7.25 times higher in those with sepsis, bleeding, and pump pocket infection compared with those with none of these complications. ${ }^{11}$ Better patient selection can lead to higher long-term survival with fewer complications and lower costs, leading to a lower ICER for the care of these patients. Readmissions are a large part of the cost of LVAD care and decrease the chance of achieving cost-effectiveness. Strategies to limit the readmission rate and outpatient costs by $50 \%$ would decrease the ICER 2.4 -fold to approximately $\$ 87,000$.

Limiting LVAD implants to situations in which they are cost-effective may be unpalatable for several reasons. First, this is the cost of progress. LVADs would not be available today if cost-effectiveness were an initial limitation. Results and cost-effectiveness have improved with better technology and patient selection, and further improvements are likely. Second, patients may live a decade or longer on device support. Unlike some expensive palliative cancer therapies in which an additional 3 months of life may define success, an LVAD may "cure" heart failure and allow the patient to experience many years of productive life. Next, the best alternative to LVAD therapy is heart transplantation, which is expensive and organ availability is limited. Finally, the precedent has been set that LVADs are an acceptable therapy, and denying coverage now would be difficult.

The challenge moving forward is balancing the use of a very expensive, lifesaving technology with the need to be good stewards of our healthcare dollars and to allocate this resource in an ethical manner. If this is accomplished, those with a good chance for success with an LVAD will have the opportunity to benefit, those with the potential for many years of productive life ahead will get the chance to reach their potential, and those who would not receive appropriate healthcare if resources are diverted to poorrisk LVAD candidates will not be denied their opportunity.

Using an ICER of $\$ 100,000$ to strictly limit implantations is not reasonable because the therapy already significantly 
exceeds this threshold. Even if the device were steeply discounted, the cost of the index hospitalization, daily care, and readmissions would still make this cost-ineffective by current standards. LVADs could be cost-effective if a high predicted long-term survival is required before implantation, but this would exclude many reasonable candidates from implantation. The best option may be to respect cost-effectiveness thresholds when possible and limit high-risk implantations to a select group of patients. Essentially, this would mean that a patient would need to fit into 1 of 2 groups to justify LVAD implantation: either low risk or high upside. In the low-risk group, LVAD therapy would be reasonably cost-effective, especially if the complication rate is low and survival for several years or more is achieved. In this group, the principle of justice would be met because these patients would not use an extraordinary amount of resources in the process. VAD risk models, although not perfect, are a useful tool in identifying these low-risk patients.

Likewise, identifying patients at high risk is not difficult. The challenge is to identify high-risk patients with a high upside who, it could be argued, would warrant LVAD implantation from an ethical standpoint based on the "rule of rescue." 12 According to this, there is often a desire to save a given patient who is facing imminent death regardless of the cost or the impact on other unidentified persons. The rule of rescue is at direct odds with costeffectiveness. The first is blind to the cost of the care or the rate of success, and the second takes both into account. ${ }^{13}$ Invoking the rule of rescue broadly to suggest that all patients with advanced heart failure facing imminent death should undergo LVAD implantation would bankrupt the healthcare system because of the high costs that would be incurred. However, would it be ethical if the rule of rescue were considered appropriate only for the small group of younger patients who were high risk for LVAD implantation? The "fair innings" argument would support an age-related differential threshold for implantation. ${ }^{14}$ The "fair innings" view supposes that each person has the right to the opportunity to experience a full life-completion of education, having a career, raising a family, and perhaps participating in the life of grandchildren. This argument suggests that a younger patient, who has not yet had these opportunities, could receive an expensive therapy that may not be available to another patient who has already had the chance to live a full life.

To satisfy the principle of justice, and thus to be ethically appropriate, cost-effectiveness should always be a goal. In the absence of a technology that meets even generous cost-effectiveness thresholds, it would be reasonable to limit implants to patients for whom there is a reasonable expectation that the cost of therapy and outcome will be average or better. Under the principle of justice, it would be ethically appropriate to deny implantation to any patient for whom the chance of success is low or the cost of the attempt would be significantly higher than average because of expected prolonged recovery or a high level of complications due to age, frailty, or comorbidities.

There will be high-risk patients who have not had the opportunity to live a full life, but have the potential to do so. In these patients, cost-effectiveness will not be achievable. In this situation, it is reasonable to proceed under the rule of rescue. However, because of the increased expense of VAD therapy under rescue conditions, this should be used under carefully vetted circumstances. Liberal use of the rule of rescue has the potential to limit care to other equally deserving patients, and thus violate the principle of justice. The indiscriminant use of the rule of rescue may lead to an occasional success, but the failures along the way cost real money, time, and effort, and are not outweighed by the occasional save from a cost-effectiveness standpoint. The physicians, as stewards of our healthcare dollars, need to try to refrain from thinking "What if we get lucky?" too often, because this approach would be cost-ineffective.

Application of this concept can be illustrated in the example given earlier, a 40-year-old patient and a 70year-old patient who will likely need short-term support before implantation of a durable LVAD, both of which are at risk for significant complications and mortality. LVAD therapy is not likely to be cost-effective in either patient, because the costs will be much higher than average and the outcomes much worse. In the case of the 40-year-old, this patient has the potential for many years of productive life ahead, especially if transplantation becomes an option, whereas the older patient is much less likely to survive many years regardless of the treatment given. The rule of rescue may be appropriate in the 40 -year-old, because this patient will face certain death without implantation, yet could live a decade or longer and come closer to achieving a full life if able to survive the operation. Implantation in the 70 -year-old may be seen as ethically inappropriate, because the chance of success is low and implantation is likely to be highly cost-ineffective. If costs decrease and outcomes improve, it may become ethically appropriate to implant an LVAD in patients such as this in the future.

When looking at the costs of care, autonomy would favor patient involvement in the decision-making process. However, there are 2 separate costs to consider: those to society and those to the patient. Certainly, the patient and family should be aware of the costs to them (both financial and nonfinancial) if an LVAD is offered so that they can decide if the personal benefit is worth the personal costs. However, patients are generally not interested in the costs to society, ${ }^{15}$ so these discussions may be inappropriate, especially if an LVAD is not to be offered. Physicians, as stewards of healthcare spending, make cost-benefit decisions on a daily basis without involving patients, such as not ordering daily chest radiographs for stable patients. Although the decision to not 
offer an LVAD is certainly larger, making treatment decisions on the basis of the cost-effectiveness is part of our duty to society and should be independent of patient autonomy when deciding on highly cost-ineffective treatment strategies.

The ethical allocation of LVAD therapy also requires a retrospective review of outcomes with an eye to the appropriateness of therapy rendered. It is not important, from a cost-effective standpoint, that a patient survives the hospitalization. Cost-effectiveness is attained through longterm survival with a good QOL and few complications. Ideally, each program would review every death that occurs within the first year, and perhaps even longer, and ask a few basic questions with the benefit of a retrospective view: Was the risk of a poor outcome elevated? Was the outcome predictable? Was this the right decision? Through a critical review of successes and failures, we have the ability to choose wisely and practice ethically appropriate medicine.

\section{Conflict of Interest Statement}

Author has nothing to disclose with regard to commercial support.

\section{References}

1. Beauchamp TL, Childress JF. Principles of Biomedical Ethics. 1st ed. New York, Oxford: Oxford University Press; 1979.

2. Stewart GC, Brooks K, Pratibhu PP, Tsang SW, Semigran MJ, Smith CM, et al. Thresholds of physical activity and life expectancy for patients considering destination ventricular assist devices. J Heart Lung Transplant. 2009;28:863-9.

3. Slaughter MS, Rogers JG, Milano CA, Russell SD, Conte JV, Feldman D, et al. Advanced heart failure treated with continuous-flow left ventricular assist device. N Engl J Med. 2009;361:2241-51.

4. Neumann PJ, Cohen JT, Weinstein MC. Updating cost-effectiveness - the curious resilience of the \$50,000-per-QALY threshold. N Engl J Med. 2014; 371:796-7.

5. National Institute for Health and Clinical Excellence (NICE). Appraising LifeExtending, End of Life Treatments. London: National Institute for Health and Clinical Excellence; 2009.

6. Special report: cost-effectiveness of left-ventricular assist devices as destination therapy for end-stage heart failure. Technol Eval Cent Assess Program Exec Summ. 2004;19:1-35.

7. Rogers JG, Bostic RR, Tong KB, Adamson R, Russo M, Slaughter MS. Costeffectiveness analysis of continuous-flow left ventricular assist devices as destination therapy. Circ Heart Fail. 2012;5:10-6.

8. Long EF, Swain GW, Mangi AA. Comparative survival and cost-effectiveness of advanced therapies for end-stage heart failure. Circ Heart Fail. 2014;7: $470-8$.

9. Baras Shreibati J, Goldhaber-Fiebert JD, Banerjee D, Owens DK, Hlatky MA. Cost-effectiveness of left ventricular assist devices in ambulatory patients with advanced heart failure. JACC Heart Fail. 2017;5:110-9.

10. Boyle AJ, Ascheim DD, Russo MJ, Kormos RL, John R, Naka Y, et al. Clinical outcomes for continuous-flow left ventricular assist device patients stratified by pre-operative INTERMACS classification. J Heart Lung Transplant. 2011;30: 402-7.

11. Oz MC, Gelijns AC, Miller L, Wang C, Nickens P, Arons R, et al. Left ventricular assist devices as permanent heart failure therapy: the price of progress. Ann Surg. 2003;238:577-83.

12. Jonsen AR. Bentham in a box: technology assessment and health care allocation. Law Med Health Care. 1986;14:172-4.

13. McKie J, Richardson J. The rule of rescue. Soc Sci Med. 2003;56:2407-19.

14. Maynard A. Rationing health care. BMJ. 1996;313:1499.

15. Irwin B, Kimmick G, Altomare I, Marcom PK, Houck K, Zafar SY, et al. Patient experience and attitudes toward addressing the cost of breast cancer care. Oncologist. 2014;19:1135-40. 